\title{
Micro-cleavages and violence in civil wars: A computational assessment
}

\author{
Conflict Management and Peace Science \\ 2016, Vol. 33(5) 539-558 \\ (C) The Author(s) 2015 \\ Reprints and permissions: \\ sagepub.co.uk/journalsPermissions.nav \\ DOI: I0.1 I77/07388942I5570433 \\ cmp.sagepub.com
}

\author{
Nils B. Weidmann
}

University of Konstanz, Germany

\begin{abstract}
Many accounts of civil war violence assume that a conflict's master cleavage also explains the local occurrence of violence. Some scholars, however, have argued that violence is often the result of local cleavages and feuds, many of which may be unrelated to the conflict's master cleavage. How is local violence related to the conflict's master cleavage? Using a computational model, this paper studies an alliance mechanism proposed by Kalyvas (2006. The Logic of Violence in Civil War, Cambridge University Press), where macro-actors support local ones that fight on their behalf. While these alliances create a principal-agent problem, the model shows that they can raise the overall severity of the conflict and serve the interests of the macro-actor. However, the model also shows that these mechanisms work only under limited conditions. Alliances can increase the level of violence perpetrated in the interest of the macro-actor, but only if (a) the latter supports agents that have in the past fought along the master cleavage and if (b) this happens in rural areas. This emphasizes again the importance of the rural dimension in the study of civil war.
\end{abstract}

\section{Keywords}

Civil war, computational modeling, micro-cleavages

\section{Motivation}

The past decade has witnessed a sharp rise in academic interest in the phenomenon of civil war. More recently, debates have emerged about the "right" level of analysis under which to study civil wars: criticizing aggregated country-level analysis such as Fearon and Laitin (2003) or Collier and Hoeffler (2004) as overly simplified and imprecise, scholars have generated new data and techniques to move the study of civil war to more precise levels of analysis (Cederman and Gleditsch, 2009). This "disaggregated" approach typically focuses on groups as conflict actors (Cederman et al., 2010), subnational conflict regions (Buhaug and Gates, 2002) or the local dynamics of violence (Weidmann, 2011).

\section{Corresponding author:}

Nils B. Weidmann, Department of Politics and Public Administration, University of Konstanz, 78457 Konstanz, Germany.

Email: nils.weidmann@uni-konstanz.de 
Yet even if these disaggregated studies represent a significant refinement in analytical resolution, they assume that civil wars typically feature dyadic actor constellations between a government and (one or more) challenging group(s). While this dyadic framework may help us explain the onset of conflict, Kalyvas (2003) cautions against using it to study the microdynamics of civil war violence. He argues that frequently, and mistakenly, we tend to apply what appears to be a conflict's macro-cleavage (e.g. political ideologies or nation wide ethnic identities) to the local level, with the implicit assumption that this master cleavage should equally explain patterns of violence at the local level. However, there is often a "disjunction between the macro-level causes of the war and the microlevel patterns of violence" (Kalyvas, 2006: 4). In other words, he advocates a more careful perspective on local cleavages and feuds, which he argues are often unrelated to the master cleavage (Kalyvas, 2003). Describing a wealth of anecdotal examples from a range of different civil wars, he claims that micro-cleavages such as "turf battles between neighborhoods", "vendettas" or "clan rivalries" substantially shape civil war dynamics.

To what extent does this local perspective on the causes of violence challenge the binary macro-perspective? We probably would not want to subscribe to the notion that civil wars are exclusively driven by local actors and their private motives, as proposed by some authors (Mueller, 2000). At the same time, however, the various anecdotes presented by Kalyvas (2003, 2006) strongly suggest that the "local" - and in particular, the disjunction between micro- and macro-cleavages - cannot be ignored. The only way forward is to find out more about the mechanism linking the two levels: what causes micro-feuds to become activated under the conditions of larger-scale, national-level civil war?

Kalyvas (2006) presents one particular mechanism to fill this gap. He suggests that alliances between macro- ("supralocal") and local actors explain how violence plays out on the ground. Supralocal actors are those fighting along the conflict's master cleavage. In order to take advantage of local networks and power, these supralocal actors support local ones with external resources, and thus allow them to gain an advantage in the local feuds they are involved in. Thus, violence in civil war serves private and political goals at the same time; it is fought by private, local entrepreneurs, but supported by national-level actors to foster a particular political project.

While intuitively appealing, the suggested mechanism needs further theoretical specification and empirical testing. Alliance formation between micro- and macro-actors may not be straightforward at all. For example, the macro-sponsor usually has little control over the micro-actions of its ally. What if violence is ultimately employed to foster the micro-entrepreneur's private motives, rather than the larger political agenda of its supporter? This situation is essentially a principal-agent problem of violence delegation (Salehyan, 2010), and can entail adverse selection and moral hazard. These problems are likely to be particularly severe in the context of ongoing civil war, where reliable information about the local actor's true motives is difficult to obtain, thus making external support particularly risky and errorprone. Is the alliance mechanism viable?

Not surprisingly, it is extremely difficult to conduct systematic empirical research that would be able to answer this question. Given the almost infinite number of micro-cleavages and -actors that could potentially explain wartime violence, a more general test of how they align with the macro-cleavage seems to be difficult at best, or even outright impossible. This is why this paper suggests a different route. By implementing a computational model with a set of micro-actors that generate violence, we are able to examine what happens if we support some of them that claim to be fighting along a particular macro-cleavage. The model 
shows that, even under relatively simple assumptions and boundedly rational violence entrepreneurs, there is evidence that conditional support exacerbates the overall generation of violence considerably. Interestingly, this effect does not require perfect information about micro-actors' motives. At the same time, the model shows - in line with the literature - that the effect seems to be limited to rural areas with tightly knit social structures.

\section{Macro-abstractions, micro-criticisms and how to marry them}

The literature often portrays civil wars as binary actor constellations, where a government fights a rebellious party that differs from it along some dimension. This dimension can be of different kinds. For example, the "Sendero Luminoso" rebels in Peru or Chairman Mao's revolution in China differed from the respective opponent side along a political left-right dimension. The Philippines and Ireland, among other cases, have seen conflict between groups of different religions, and in Bosnia, Georgia and Turkey, ethnicity is the master cleavage that distinguishes the fighting camps. Almost every conflict has its perceived macro-cleavage that separates one side of the conflict from the other. It is an (often implicit) assumption in many analyses that civil war violence at the local level is perpetrated across this macro-cleavage. For example, the Armed Conflict Locations and Events Dataset by Raleigh et al. (2010) - a data collection that includes individual violent incidents in civil wars - codes civil war events exclusively as occurring between the government and a rebel group, thus imposing a dyadic actor constellation that supposedly translates to all civil wars. Analyses using this type of data necessarily rely on the same abstraction; see for example Raleigh and Hegre (2009), Hegre et al. (2009) or Weidmann and Ward (2010). More recent data collection projects, such as the Uppsala Conflict Data Program's Geo-referenced Event Data Set (Sundberg and Melander, 2013), follow a similar approach.

Kalyvas (2003) was one of the first to criticize the mismatch between this assumption and the messy realities of civil war. He argues that violence in civil wars is frequently unrelated to the master cleavage; rather, civil wars create an environment that permits the settling of local cleavages and feuds by violent means. Often these micro-cleavages existed before the war started, and they frequently have little relationship to what the war was about. Micro-cleavages can be of different types, and Kalyvas (2003) presents a large number of anecdotes illustrating this. One example is the case of a Spanish village during the civil war in the 1930s, where a "dispute between two doctors competing for the title of official town doctor" led to violence between people siding with each of the doctors (Kalyvas, 2003: 478). As another example, he mentions that the mass killings in Indonesia in the 1960s did not match the conflict's master cleavage (communism/anticommunism), and frequently were the result local conflicts, for example, between settlers and locals, or Protestants and local religious groups. The observation of the local nature of cleavages in civil wars is not only present in Kalyvas's writings, but has also been mentioned in other work (see e.g. Fujii, 2009 on the conflict in Rwanda).

Is violence exclusively local, as some authors suggest (Mueller, 2000)? If it is, overall levels of violence should be comparable to peacetime, which they are clearly not. Rather, the common perception is that civil war activates local feuds that would otherwise have gone unsettled. Thus, conflict along a master cleavage is a coordinating device that allows private actors to use violence for their private means. In short, political violence activates private violence. Yet how exactly does this happen? In order to make progress in the study of civil war, we need theories that allow us to bridge micro- and macro-levels. In particular, we need 
to analyze the mutual dependence between the macro- and the micro-level, as neither alone can fully explain the dynamics of violence on the ground.

One mechanism that is able to do so has been proposed by Kalyvas (2006). He argues that alliances between macro-level organizations and micro-level conflict actors are the theoretical mechanisms linking the two levels. Supralocal or macro-entrepreneurs supply local actors with resources, such that the latter become more powerful in local disputes. However, this support is conditional on the local actor fighting - at least to a large extent - in the interests of their external sponsor. So in essence, this setting entails a principal-agent problem, where the macro-actor outsources the generation of violence to the local one. However, even if this brings with it some advantages for the former, such as access to local networks, there are also significant drawbacks. For example, the true interests of a local actor may only partly overlap with those of the macro-actor. The risk in supporting the local actor is that it will use the means provided exclusively to pursue its own ends. Of course, the local actor will be hesitant to transform its own struggle into one related to the master cleavage, as it would lose its own significance and become only an instance of the struggle at the national level.

The obvious way for the macro-actor to avoid this is to support exclusively those conflict entrepreneurs whose motives seem to overlap considerably with their own. This, however, is difficult to assess, for a number of reasons. First, local information is difficult to obtain, in particular in times of conflict. For the same reason that the macro-actor intends to hire the local actor - to tap into local networks - it may become difficult to assess whether the latter would be a reliable partner in an alliance. This problem is known as "adverse selection." Second, even if it were known which side in a local dispute an actor in question is fighting on, this dispute may be orthogonal to the larger, national-level issue, and thus provide no information as to the stance the actor will take in the latter. This entails the potential for moral hazard during the conflict. Third, all of this is complicated by the fact that local actors and feuds may be fluid and change as a result of ongoing violence, which makes a good assessment as regards the true nature of a local ally much more difficult.

All this leaves the question of whether the proposed mechanism of alliance formation is indeed a feasible link between the micro- and the macro-level. In order to find out, this paper presents an agent-based model of micro-level violence in civil wars. Actors are involved in private disputes at the local level. The purpose of the model is find out how the introduction of external support for these actors along a particular macro-cleavage changes the dynamics of violence, in particular its overall level. In particular, the purpose of the model is to analyze how a fuzzy outside assessment of local actors - which, according to the above theoretical considerations, should render the alliance mechanism less powerful-really plays out. In implementing such a model, we have to solve a series of modeling challenges.

First, we need to represent different potential cleavages in the agent population. Individuals differ along many dimensions, such as political orientation, ethnicity and religion. Some of these characteristics could emerge as cleavages along which violence is perpetrated. Yet, the weight of these dimensions in a conflict could vary considerably, not only between conflicts but also within. For example, religion could be the dividing line in one conflict region, but not in another (see the Indonesia example above). This is essentially the micro-cleavage argument discussed above, where it is not the master cleavage but some local differences that trigger violence. Therefore, we need to incorporate the fact that different individuals may hold different social categories responsible for violence, and that the weight of these cleavages in generating violence could vary among the population, also through endogenous effects. 
Second, as many of the presented anecdotes show, there is often considerable uncertainty about the nature of violence. If two individuals differ both along the master cleavage but also along a micro-cleavage, how can we know what triggered an act of violence between these individuals? This uncertainty often affects observers of civil war violence, and has implications for how they interpret the nature of violence. However, continuing exposure to, and observation of, civil war violence will lead individuals to form beliefs about the nature of the conflict. The model therefore incorporates uncertainty about both the nature of violence and the updating of beliefs.

The resulting model is one that predominantly focuses on the micro-generation of violence by local conflict actors. These actors exclusively fight for their own private motives. Alliance formation is implemented simply as provision of external resources - in other words, making some of the local actors stronger than others. As such, the model represents a "lower boundary" of the effect of micro-macro-alliances. If alliances work under this highly simplified scenario that is heavily biased toward the local, we should expect them to have an even stronger effect in a more realistic scenario, where for example strategic local entrepreneurs are able to adjust their behavior if promised external support.

\section{A computational agent-based model}

Because of the motivation outlined in the previous section, we resort to a technique called agent-based computational modeling to create a simple model of cleavages and violence in civil wars. In an agent-based model, the researcher can "create, analyze, and experiment with, artificial worlds populated by agents that interact in non-trivial ways and that constitute their own environment" (Cederman, 2001: 16). The feature that distinguishes agentbased models from other types of computer modeling is that agents are represented as individual computational entities possessing certain characteristics, but also follow certain behavioral rules to interact with each other. This type of simulation is particularly suited to modeling social phenomena where we observe society-wide patterns that emerge as a result of the interactions of individuals. The following sections introduce a model of micro- and macro-cleavages in civil wars. Although the model is rather simple, the paragraphs that follow focus on conveying intuition rather than describing the model in detail, which is done in the Online Appendix.

\section{Actors and their characteristics}

The core components of the model are individuals that engage in violence. In order to move away from a simplistic binary conception of the parties in a civil war, we let these agents differ along a set of dimensions rather than a single one. In other words, rather than assuming a single macro-level cleavage such as ethnicity, race or political orientation, agents possess all of these characteristics at the same time. However, as discussed below, not all of them have equal salience at any point in time, so an agent could be part of, for example, an ethnic group without this having any impact on agent-to-agent relations. The model follows earlier attempts at representing the multidimensionality of societies by assigning each agent a sequence of zeroes and ones that represent agents' characteristics along a set of dimensions (Axelrod, 1997; Lustick et al., 2004). For example, assuming five dimensions, an agent could be assigned the string 01100, where the first dimension could, for example, represent 
political orientation, and the zero along this dimension could indicate that the agent is a member of the Marxist camp.

This particular representation does not require us to assign a particular meaning to each of the dimensions; all it assumes is that individuals have a set of individual characteristics, and that any two individuals could be similar along some dimensions (i.e. belong to the same ethnic group or the same political camp), but at the same time differ along others. As desribed above, agents do not consider all social dimensions to be relevant at the same time. Rather, they emphasize certain dimensions over others. For example, an agent could consider ethnicity to be the most important dimension (and behave accordingly), whereas another places more emphasis on political orientation. The relative importance of social dimensions to an agent is modeled as a (discrete) probability distribution over the set of dimensions. Each agent keeps its own importance distribution. At the initial stage of the model, this distribution is uniform for all agents; however, as we will see below, it subsequently changes as a result of conflict.

In addition to their social characteristics, agents are equipped with a "resource" counter. An agent's resources decrease whenever it is involved in violence, which is described in more detail below. This counter is simply meant to capture the fact that perpetrating violence has certain costs to an agent, and thus cannot be done solely at the agent's discretion: if an agent has no resources at its disposal, it cannot initiate violence. Resources are implemented in the model as a positive real number per agent, which is initialized with a random level from [1, 10]. After each step in the model, each agent receives one new resource unit. This "uniform replenishment" is the default scenario of the model, but we will consider alternative implementations when analyzing micro-macro-alliances below.

\section{The social structure}

Agents in the model are connected by a network that forms the social structure of the population (or in agent-based model terminology, the "interaction topology"). Rather than resorting to a static grid as is done in many well-known computational models (Schelling, 1978), we operationalize the interaction topology as a network of links between agents. The network defines which agents are "socially proximate" to a particular agent, and thus constitute possible interaction partners. An agent's neighbors are those that are connected to it in the network. These neighbors are possible opponents in the simulation, that is, those that are directly involved in violent interactions, but are also observers of these interactions.

The network structure employed in the model is based on a "small-world" network proposed by Watts and Strogatz (1998). A small-world network captures different empirical properties that have been found in real-world social networks. Most importantly, a smallworld network exhibits high degrees of clustering as in typical social networks: clustering indicates that somebody's acquaintances are often also mutual acquaintances, as is frequently the case in tightly knit communities. At the same time, a small-world network can parametrize the extent to which this is the case; therefore, it can represent a "rural" structure that consists only of villages with tight intra-village connections, but few inter-village links, but also an "urban" one where the social environments of people overlap to a large extent. This urban-rural dimension is represented in the model using an urban parameter $u$ from $[0,1]$, where low values generate a rural network (largely disconnected villages), and high values correspond to an urban one. ${ }^{1}$ 


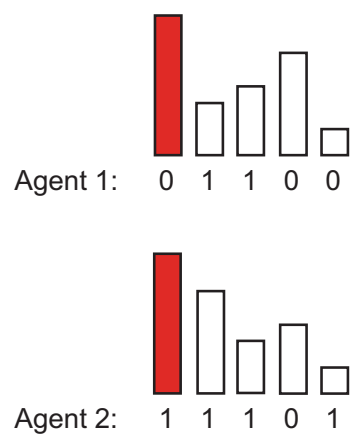

Figure I. Example demonstrating the encoding of social dimensions and the importance attributed to them by the agents. Each agent is characterized by a set of bit strings of length 5 . Different numbers along one dimension indicate that the agents belong to different "sides" for this particular dimension, as is the case for the first and the last dimension in the example. In addition, agents believe some of these dimensions to be more important than others. This is indicated by the bars in the example. However, a difference along a particular dimension does not necessarily mean that it matters to the agents; this is only the case if both agents perceive the dimension along which the difference occurs to be salient.

Note that the number of links in these networks is the same irrespective of the value of $u$, which is important to ensure that any differences in outcomes found across the range of $u$ are not simply due to different numbers of links but rather their structure.

\section{The occurrence of violence}

The model simulates the occurrence of violence between agents. Note that we are not interested in whether violence occurs at all; rather, we measure differences in the severity of violence under different conditions. Also, the particular direction of violence is not of interest here, so there is no distinction between the initiator of violence and the target. Rather, the model is intended to simulate under which circumstances higher levels of violence are generated, and what different mechanisms of micro-macro-alliance formation contribute to that.

In the model, violence occurs at the dyad level. That is, at every time step during the simulation, a random link in the network is selected, and violence occurs under certain conditions. The decision whether this happens is contingent on two factors: motivation and opportunity. Motivation essentially captures the willingness of the two agents to use violence, and opportunity captures whether they have the means to do so.

The basic motivation for violence between two agents is the perceived difference between them. Perceived distance along one dimension is high if (a) both agents are part of different "sides" for a particular dimension (i.e. one has a 0 and the other has 1 in the respective dimension), and (b) if both consider this dimension to be salient (i.e. the importance distributions of both agents have high values for the particular dimension). Figure 1 illustrates this for an example with five social dimensions. The total perceived difference is simply the sum of the differences over all dimensions, which results in a motivation score between 0 and 1 (see Online Appendix for details).

The second factor determining whether violence occurs is opportunity, that is, the means that agents have to perpetrate it. These means are captured by the agents' resource levels. The opportunity for violence increases the weaker one of the agents is in comparison to the 
other. The logic behind this is that opponent strength deters: if facing a strong opponent, agents are simply afraid of the damage that could occur to them, and thus refrain from engaging in violence. In the model, opportunity for conflict along a dyad is calculated on a scale between 0 and 1; it takes a value close to 1 if the weaker agent has almost no resources as compared with the other, and gets close to 0 in the case of resource parity.

For violence to occur, both motivation and opportunity have to be high (Starr, 1978). There will be no violence if agents simply do not perceive themselves to be sufficiently different for there to be a reason to fight. However, even if there is a difference, the resources they have at their disposal need to permit them to engage in violence. Thus, the decision whether violence occurs is simply a multiplication of the motivation and opportunity scores, each computed as described above. This will result in a "violence risk" score, a number from $[0,1]$ that takes a high value if both motivation and opportunity are high, but a low one if at least one of them is low. The model then simply makes a random Bernoulli draw, such that, with a probability equal to the risk score, violence occurs along a particular dyad.

\section{Direct and indirect effects of violence}

Violence, if it occurs, has two effects: direct effects on the agents engaged in it, and indirect effects on the observers of a particular episode. The direct effects are straightforward: in line with assumptions made in similar types of conflict models (Cederman, 2003), the damage that agents inflict on their opponents is proportional to their strength. In other words, stronger agents decreases their opponents' strength more than weaker agents do. Once violence occurs in the model, the resource level of a participant in a particular episode simply decreases by $30 \%$ of its opponent's resources. If the decrease is larger than the agent's resource level, the agent dies and is replaced with a randomly initialized new agent at the same network position. Thus, agent regeneration does not change the network structure, but only replaces a particular agent.

An incident of violence between two agents also has indirect effects on the immediate social neighbors of the two agents involved. The basic logic behind this is that the occurrence of violence has a signaling effect as regards the society's most salient social dimensions. If, for example, violence occurs frequently between members of two different ethnic groups, this will inevitably lead to a stronger, and much more pervasive, salience of the ethnic dimension in a society. In other words, ethnic violence will lead the observers of this violence to reinforce the ethnic dimension in comparison to others, or in the language of the model, to shift importance from other dimensions to the ethnic one.

In reality, however, it is often difficult to observe the nature of a particular incident. As many anecdotes from the civil war literature demonstrate, different observers sometimes interpret the nature of violence in different ways: was an incident due to ethnic differences, or political ones? What is only visible is the opponents' membership in different ethnic, political or religious camps. Therefore, the model makes the assumption that the only information available to outside observers of violence is the opponents' values along the social dimensions (i.e. the information contained in the $0 / 1$ string), but not the importance that each opponent attributes to the dimensions. So in short, observers will only see whether a particular incident occurred, for example, between members of two ethnic groups, but not whether the opponents were fighting for reasons related to their ethnicity, or some other personal dispute. 
Absent any detailed information about the nature of a particular incident, the observing agents assume that any dimension along which the two opponents differ could be responsible for it. Thus, they shift importance to all those dimensions where the two opponents are different, that is, where their $0 / 1$ strings have different values. More precisely, the new importance distribution after observing violence is simply the average of the old one the agent held previously and the new one (which represents those dimensions where the two agents differ). This ensures that prior experience and newly observed information have equal weight.

\section{Summary of model dynamics}

This section provides a short overview of the model dynamics. At the beginning of the simulation, a number $N$ of agents are created. These agents are initialized with random $0 / 1$ strings of a certain length (the "number of dimensions"), and with random strength levels from [1,10]. A small-world network with parameter $u$ is created between the agents. As with most agent-based simulations, the model then iterates through a number of time steps. In each time step, $N$ network links are randomly selected and activated in a random sequence. For each of these activated dyads, the model then:

1. computes the violence motivation by summing up the multiplied importance values of the agents along the dimensions where the agents differ

$$
R_{\min }+R_{\max }
$$

2. computes the opportunity for violence as

$$
1-\frac{R_{\min }}{R_{\min }+R_{\max }}
$$

where $R_{\min }$ is the resource level of the weaker agent and $R_{\max }$ is the resource level of the stronger agent;

3. computes the conflict risk as the multiplication of motivation and opportunity;

4. with probability $=$ conflict risk, lets violence occur along the dyad.

If violence occurs,

- the opponents' strength levels decrease by a proportion of the other's strength; and

- each agent that is connected to the two participants shifts its importance distribution to those dimensions where the two fighting agents differ.

At the end of each time step, each agent receives one unit of resources, so a total of $N$ resource units are distributed. This resource replenishment is the default behavior, which will be modified below when implementing different alliances.

\section{Experiments and results}

The purpose of the model is to explore how micro- and macro-level cleavages jointly affect the dynamics of violence on the ground. In particular, we are interested in whether a simple mechanism of alliance formation increases the intensity of conflict, as hypothesized in the literature. To this end, the model presented above is implemented in NetLogo as a 
computational agent-based simulation. NetLogo is a simple yet powerful simulation toolkit for agent-based models (Gilbert, 2007; Wilensky, 1999), and is available free of charge for all major operating systems.

The experiments start with an exploration of the baseline model that focuses only on local dynamics. Having explored the nature of violence in this model, we then implement a simple mechanism of alliance between macro-actors and micro-level violence entrepreneurs. The core idea of this alliance mechanism is that the former support the latter with resources for fighting, but only if the latter fight for similar ideals. In practice, however, these ideals are often not clearly observable, and macro-level entrepreneurs face a risk of selecting microactors that ultimately will not support the macro-actor's cause. In the experiments, we will explore different ways by which macro-actors can select their local allies. In particular, we will ask the question how much the former need to know about the latter to make alliances successful, and whether we can assume this knowledge to be present in real-world cases.

Owing to the number of parameters in the model, it is necessary to devise a strategy by which the model's parameter space is to be analyzed. The standard way of doing this is to define ranges for the parameter values of interest, and then traverse the multidimensional parameter space systematically (in so-called "parameter sweeps"). With more than a couple of parameters, this strategy quickly becomes infeasible owing to the high number of runs required. This usually leads authors of agent-based models to narrow down the parameter space by shortening the ranges of parameter values, and to test only a few selected combinations of them. This is unsatisfactory, as large parts of the parameter space can be left unexamined. For that reason, we implement a different strategy here. As Laver and Sergenti (2007) argue, one way to alleviate this problem is to sample randomly from a larger parameter space. In other words, with broad ranges of values defined for each parameter, a large number of simulations is run with randomly drawn parameter vectors from this space. This way, we avoid having to restrict the model runs to narrow ranges of values, and/or certain combinations. In addition, this Monte-Carlo experiment ensures a uniform distribution of runs across the parameter space at all times, which means it can easily be distributed across different machines. It also eliminates the need for averaging results across multiple runs with different random seeds. For the model analysis in this paper, a large experiment with 100,000 runs was conducted, and all results reported below are based on this experiment. The descriptions below list the parameter ranges used for this.

\section{Experiment I: local entrenchment}

What influences the level of violence in the simple model outlined above? We first explore the model using a baseline scenario without alliances, and examine in particular the impact of urbanization, which is the main parameter of interest in this model. A number of parameters are not of primary theoretical interest here, so we draw random values for them according to the Monte-Carlo procedure outlined above, with the ranges specified as listed in Table 1.

The violence-generating process in the model strongly focuses on the micro-level. That is, violence is exclusively generated during interactions between micro-level actors. These violent interactions are the result of perceived social differences between actors, but in turn also reinforce these differences through a signaling effect that violence has on observers. More precisely, violence along a particular social dimension should strengthen the importance of this dimension among the immediate "bystanders", which in turn will cause them to use 
Table I. Parameters and ranges used in experiment I. Main parameter of interest in bold

\begin{tabular}{ll}
\hline Parameter & Range \\
\hline Level of urbanization & Real number between 0 and I \\
Number of agents $N$ & Integer number between 100 and 500 \\
Number of social dimensions & Integer between 5 and 10 \\
Small-world network connection radius & Integer between 3 and 8 \\
\hline
\end{tabular}

violence along the same dimension. Thus, what should happen in the model is an entrenchment of local divisions, where at the local level, certain dimensions matter more than others, and neighbors share a common focus on these dimensions. In other words, there should be local conflict cleavages that emerge at the local level, but these cleavages may not necessarily be the same across villages.

Can we observe local entrenchment also in the model? As a first test, we analyze how the urban-rural dimension affects similarity of agents in terms of ideology. In the model, this similarity manifests itself if two agents consider the same dimension to be most important, or in other words, if the modal values of their importance distributions are the same. If the above expectations are correct, local entrenchment should occur in rural settings where people are usually part of a single, dense local network. Initial results clearly provide evidence for local entrenchment. Figure 2 (left) shows that, as we increase the urbanization parameter from 0 to 1 , the average proportion of like-minded neighbors (those that consider the same dimension as most relevant) decreases from about 0.6 to 0.2 . So in other words, in rural settings roughly $60 \%$ of an agent's neighbors share its ideological beliefs, while in urban areas this is the case for only $20 \%$. Thus, in rural areas, local camps of agents form; through violence along particular cleavages, these camps develop their own enmities and feuds.

The fact that local entrenchment occurs does not mean that it affects the overall level of violence. In order to find out, we measure the average rate of attacks (along an activated dyad) during the simulation run. Note that all runs last exactly for 1000 time steps, which is when the model has had sufficient time to converge to a stable rate of violence. ${ }^{2}$ Figure 2 (right) shows that local entrenchment has a visible effect on the level of violence. In rural areas, the conflict rate is as high as about $6 \%$. In urban environments, this level drops to a rate of about $5.5 \%$. Clearly, local entrenchment contributes to the creation of local hotbeds of violence, which raise the overall intensity of the conflict.

In sum, this first experiment provides some interesting insights. As explained above, violence leads to a local entrenchment of cleavages, where local disputes reinforce and strengthen themselves. Thus, the experiment illustrates that cleavages are endogenous to the conflict; they emerge as a result of violence, but also influence subsequent action. While this is not too surprising given the design of the model, results show that local entrenchment is more likely to work in rural areas. Thus, the structure of the social network determines where it can take root. At the same time, there is no convergence toward a single master cleavage, since all of these local disputes are about different issues. At least in part, this setup resembles the many anecdotes that emphasize the local nature of violence in civil wars. However, in our baseline experiment, violence is exclusively local and no coordination occurs along a master cleavage. In the following experiments, we will explore if and how a simple alliance mechanism around a particular cleavage affects conflict dynamics. 

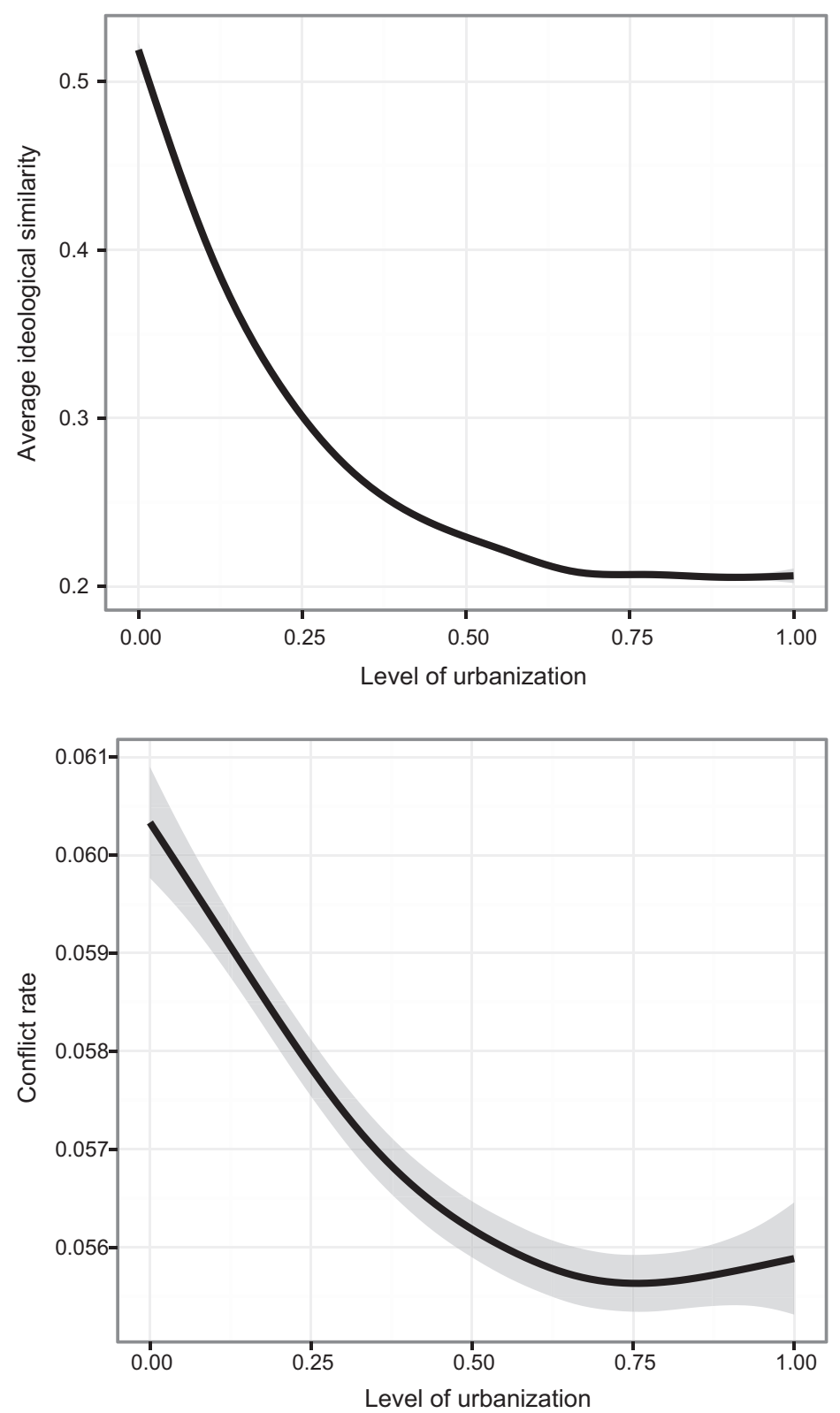

Figure 2. Effect of the urban-rural dimension on local entrenchment (left) and conflict severity (right).

\section{Experiment 2: micro-macro-alliances and the severity of conflict}

As outlined in the theory section, Kalyvas (2006) sketches a mechanism - alliance - that connects micro-level actions of conflict entrepreneurs with macro-level organizations. An alliance, according to Kalyvas, serves both sides: on the one hand, by providing local actors 
with external material support, macro-actors are able to tap into local networks and generate violence. On the other hand, local actors take advantage of this support, and can fight for their own private motives, which they otherwise would not be able to do.

In order for their material support to be invested well, macro-actors need to ensure that local actors fight, at least partly, in their interest. Macro-actors face a risk that resources spent on the generation of violence result exclusively in the settlement of local feuds, which have little to do with the master cleavage. This situation is essentially a principal-agent problem: macro-actors hire local ones to fight, but they have only limited control over the extent to which violence is actually employed in their interests. So, for example, if a local actor receives support for carrying out attacks against a particular ethnic group, but uses these resources only to fight a family feud, from the perspective of the macro-actor these resources are not well invested.

In this second experiment, we develop and test different macro-micro-alliance mechanisms. These mechanisms differ in the extent to which a macro-actor can observe the true motives driving a local actor to use violence. Ideally, in order to ensure true commitment, the macro-actor needs to observe the inner motives of a local actor, which is usually difficult, if not impossible, to gage from the outside. This is why we will examine a range of mechanisms, from ones based on publicly visible information on a local actor to those taking into account the inner motives of the local actor. Overall, examining the range of these mechanisms allows us to do two things. First, it allows us to test whether alliances are catalysts of violence and let local feuds crystallize around macro-cleavages. Second, it helps us assess the plausibility of the alliance mechanism: if alliances only work if the true motives behind the actors can be perfectly observed (which is not a plausible assumption), this would cast doubt on the alliance mechanism in general.

Before introducing the different mechanisms, let us take a look at how alliances in the model work in general. We implement alliances as selective support from a macro-actor for a subset of the population of local agents, and the different mechanisms only differ as to how the supported agents are selected. More precisely, alliances are implemented as follows. Recall that, in the baseline model, each agent receives one unit of resources at the end of each time step. In order to model a support mechanism through an alliance, we change the default resource replenishment such that a subset of all the agents selected by the macroactor receives a higher amount of resources. At the same time, in order to keep the total amount of resources equal to the baseline model, the remaining units of resources are distributed equally to the other, non-allied agents. This implementation ensures that the total amount of resources that enters the simulation is the same as in the baseline model; the only change we make is that we move from a uniform allocation of resources to agents to a skewed one, where the allied agents get more. Note that, because the subset of allied agents is selected again and again during the simulation, this mechanism allows for the withdrawal of support for agents that do not behave as expected.

Let us assume that, without loss of generality, a macro-actor intends to generate violence along the first social dimension. In other words, this actor supports local agents that appear suitable alliance partners when it comes to generating violence along the first social dimension. Let us assume further that the macro-actor belongs to one side along the first dimension, for example, " 0 ". If, for example, the first dimension corresponds to ethnicity, and " 0 " in this dimension corresponds to one particular ethnic group, this would mean that, through means of local alliances, a macro-actor aims to generate violence involving this group. Recall that membership of agents in particular categories is determined randomly, so with a focus 
on the " 0 " camp we are not biasing our results in any way, and could run the same experiment with a focus on another dimension and category.

Now, how should the macro-actor select the local agents she supports? Ideally, the macroactor would want to support those agents that are likely to be fighting for the right cause in the future. Yet how can we gage this? In the following, we introduce three heuristics for selecting local alliance partners, which depend on increasingly difficult assumptions.

- A: No Information. The simplest heuristic for selecting alliance partners is to simply choose some that belong to the group to be supported. In our model with its focus on the first dimension and the " 0 " side, a macro-actor would simply support a randomly chosen set of agents that belong to this side. This way of selecting local partners is easy to implement; membership in groups is mostly observable without major problems, and is thus public information. However, the limitation of this procedure is that membership in groups does not guarantee commitment to the group's cause; while being a member of a group, a local actor could attribute very little importance to this social dimension, thus failing to act on its behalf.

- B: Observed Behavior. A slightly more complex selection mechanism is based on observed behavior of a local agent. If an agent has been involved in violence along the macro-cleavage (dimension 0), it is more likely to have a higher motivation along this dimension as well. This, however, is not a perfect assessment of the agent's motive, since the agent may have been fighting because of other, private feuds, and the resulting violence may simply appear to be related to the macro-cleavage.

- $C$ : Internal Motive. Finally, we test the (at least, theoretically) optimal alliance mechanism where the macro-actor can perfectly observe the true motives of each potential local ally. In the model, this means that the alliance partners are selected based on their group membership ("0" in the first dimension) and the value of their importance distribution for the first dimension (with higher values corresponding to more attractive alliance partners).

In this experiment, we will examine the impact of alliances on the overall level of violence in the simulation. We keep the Monte-Carlo approach and the parameter ranges from experiment 1 but, in addition, implement the three alliance types as follows. As described above, these types only differ with respect to the selection of agents allied with the macroactor. This is the main experimental variation we are interested in. In addition, we need to define for all alliances (a) their size, that is, the proportion of the agent population that is supported by a macro-actor, and (b) the level of support that each member of the alliance obtains from the macro-actor. The latter is given as a multiple of the basic support level implemented in the base model, where each agent receives one unit. Thus, an alliance support level of 2 would mean that each alliance member receives twice this level. Size and level of support are not of primary theoretical interest, so we vary them again within reasonable limits to exclude any systematic effect of particular values. Table 2 shows the additional parameters used in the Monte-Carlo experiment.

In order to test how alliances affect violence in urban and rural areas, we measure the average rate of conflict for each type of alliance and compare it with the average rate when no alliances are used. We do so across different levels of urbanization, as in experiment 1 above. Figure 3 shows the results of this exercise. 
Table 2. Parameters and ranges used in experiments 2 and 3. Main parameter of interest in bold

\begin{tabular}{ll}
\hline Parameter & Range \\
\hline Alliance type & A, B or C \\
Proportion of allied agent population & Real number between 0.1 and 0.3 \\
Level of support for allied agents & Real number between I.5 and 3
\end{tabular}

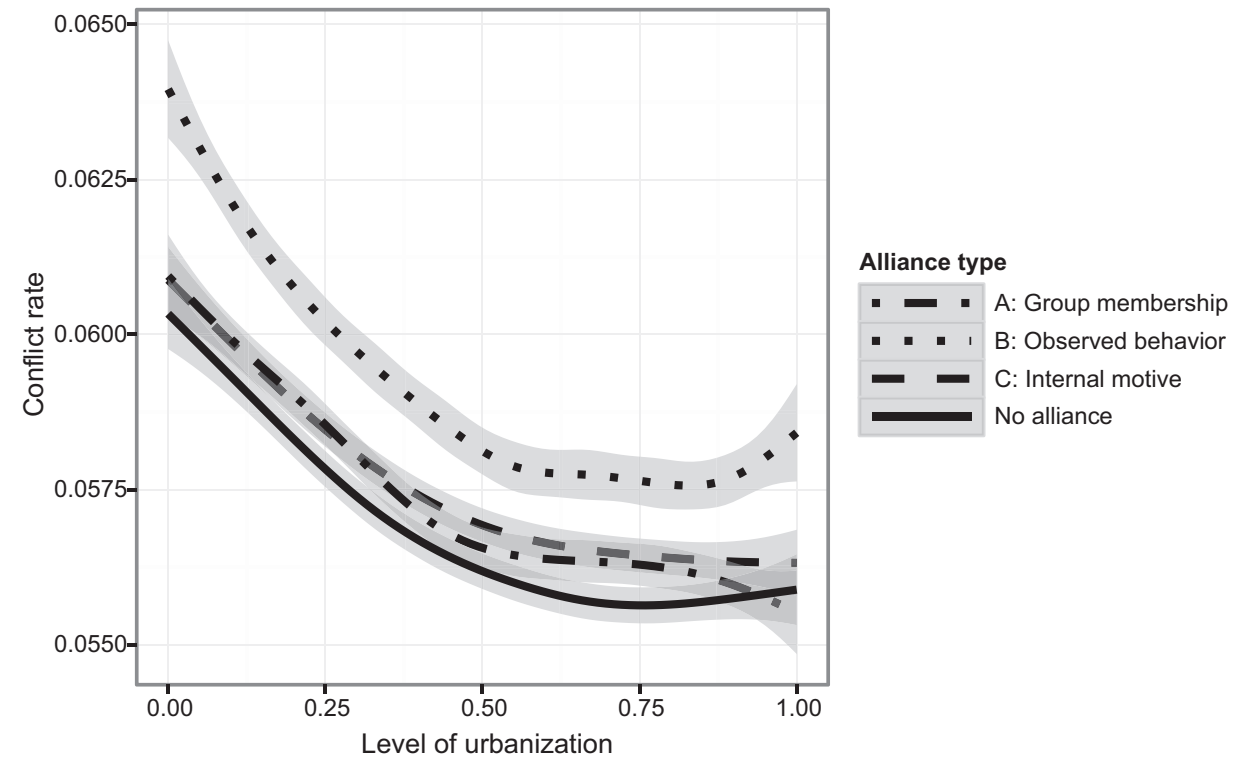

Figure 3. Effect of alliance type on overall conflict severity.

The most important result shown in Figure 3 is that alliances in general seem to aggravate violence in a civil war: the severity of the conflict increases in runs with alliances as compared with those without alliances (solid line). This is an interesting finding in itself; as described above, the total level of resources entering a simulation does not differ between runs with and without alliances. Thus, it matters how resources are distributed: the selective support of agents in the population at the expense of others can drive up levels of violence. Clearly, one particular type of alliance seems to be most conducive to this outcome, namely those based on observed behavior. The other types of alliance do increase levels of violence, but to a much lesser extent. There are few differences when it comes to the effectiveness of alliances across urban or rural areas: the positive effect on violence seems to operate throughout.

As the above experiment has shown, micro-macro-alliances foster the intensity of conflict. One reason is that the promotion of violence along a particular cleavage is a self-reinforcing spiral: once there is a slightly higher inclination to use violence along a particular cleavage, this will lead to local entrenchment where local interaction crystallizes particularly around this dimension. Therefore, a particular dimension can become a focal point around which 
the dynamics of violence evolve in a civil war, even if there is initially no particular bias towards it in the population. In all, the experiment shows that forging alliances can lead to higher levels of violence in a conflict. However, is this what macro-actors should primarily be interested in? The last experiment shifts attention to a different type of outcome.

\section{Experiment 3: impact of alliances across rural and urban areas}

So far we have only looked at one possible metric of "success" of alliances, which is the overall level of violence in a conflict. However, macro-actors should be more interested in financing violence that fits their goal, that is, that is directed against a particular group (in our model, those agents defined by a 1 in the first dimension). This is why a more appropriate way to evaluate whether alliances work would be to look at only those attacks that involve two agents that differ along the first dimension, that is, the subset of violence that is, or appears to be, related to the macro-actor's goal. Therefore, in this experiment we use the same setup as in experiment 2 above, but record the level of violence along the macrocleavage rather than the overall level. Figure 4 shows the resulting levels of macro-violence for the three alliance types across different levels of urbanization, again indicating the level of macro-violence with no alliances as a solid line.

Figure 4 shows a dramatically different picture as compared with the previous experiment. Two types of alliances decrease the level of violence that the macro-actor wants to generate, and are clearly counterproductive to its goals. Why is this so? The reason for the poor performance of the first type of alliance is simple. Selecting partners based on group membership only may be too simple a strategy, since they may not be truly committed to the goal of the group and thus not be willing to engage in violence for its goals. Observing behavior in

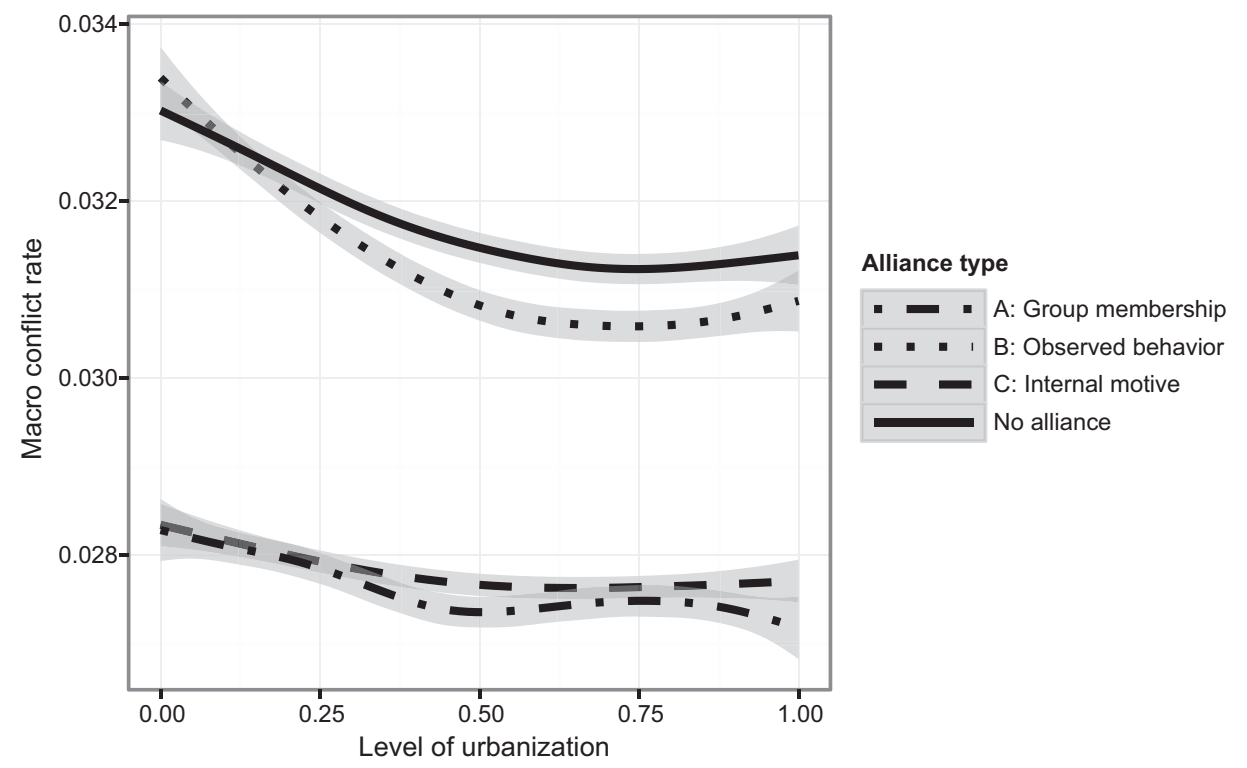

Figure 4. Effect of alliances on conflict severity, across rural and urban areas. 
addition to group membership lets the macro-actor infer this commitment without actually being able to see the motivation behind it, which, as Figure 4 shows, makes an alliance a successful enterprise from the macro-actor's point of view. However, making the true motives of a local actor available to the macro-one (type $\mathrm{C}$ alliances) - thus enabling the most accurate assessment of the former's motives - does not seem to make alliances more effective. Quite the contrary, it seems to decrease the level of violence perpetrated along the macro-cleavage.

However, how do different alliance types play out across rural and urban areas for the generation of macro-violence? Figure 4 clearly shows that type B alliances based on observed behavior can indeed be beneficial to a macro-actor, but only in rural areas. In these areas, alliances based on observed behavior are able to generate more violence as a simulation without alliances. This advantage of micro-macro-alliance disappears once we move to more urban areas; for urbanization values of 0.2 and above, the level of macro-violence generated by type B alliances drops below the level of violence in the baseline model with no alliances. Thus, alliances can help macro-actors, but only if they are based on observed behavior and implemented in rural areas.

\section{Discussion}

There are three main observations that we can draw from the results presented above. First, micro-violence enforces, and is itself reinforced by, local level cleavages. The local entrenchment we have observed in experiment 1 makes micro-violence most severe in rural settings. This suggests, conditional on the assumptions of the model, that we should see instances of micro-actor-generated violence primarily in rural areas, where dense local networks allow for local entrenchment to happen. Second, micro-macro-alliances in general have a violenceincreasing effect. Therefore, if macro-level entrepreneurs are interested in ramping up violence and generating unrest, they can do so through the selective sponsoring of micro-level actors. This suggests that, according to the model, the alliance mechanism proposed by Kalyvas (2006) looks feasible; using alliances, we can generate violence indirectly through local agents. Third, not all types of alliance work equally well when we look more narrowly at violence along the master cleavage. In fact, the alliance mechanism proposed in the literature does not discuss the principal-agent problem that arises for the macro-level entrepreneur when selecting local actors: the latter may welcome the external support, but then use it to settle their own private enmities. The results show, however, that selecting local allies that have fought along the master cleavage in the past indeed leads to a higher severity of macroviolence, and therefore to a more desirable outcome from the perspective of the macro-level entrepreneur. Thus, it seems possible to overcome the principal-agent problem using a simple and plausible way of selecting local allies. Again, however, this is limited to rural areas; as the simulations show, behavior-based (type B) alliances do not lead to the desired outcome if implemented in cities.

Where do we go from here? It would of course be desirable to subject these stylized model predictions to an empirical test. However, this is difficult; prior to the occurrence of violence, we may have little theoretical guidance of the possible local cleavages that turn violent. Therefore, we cannot map out the universe of local cleavages across different cases, in order to find out which of them actually did turn violent. However, we can use the anecdotes presented in Kalyvas (2006: chapter 11) to illustrate a basic patterns also found in the model: the instances of "local", micro-violence described in this chapter are almost 
exclusively rural; they often describe instances where local feuds among peasants was mistakenly framed as violence owing to the conflict's master cleavage. Thus, the model's prediction that the micro-violence it generates occurs primarily in rural areas seems to bear out, at least anecdotally. Unfortunately, however, there are not enough anecdotes described in the chapter that specifically look at micro-macro-alliances, so a similar plausibility check is not possible for the effect of alliances and their different types.

\section{Conclusion}

Macro- and micro-level research on civil wars is currently progressing largely independent of each other. Micro-researchers accuse macro-approaches of tremendously oversimplifying the nature of violence on the ground, and macro-researchers counter by questioning the relevance of private, local motives that are supposedly driving the dynamics on the ground. Following Kalyvas's (2006) call for mechanisms linking both levels, this paper aims to provide an assessment of the proposed micro-macro-alliance mechanism. Departing from an entirely local generation of violence generation, it shows that alliances implemented as support conditional on certain private motives can serve as a catalyst to conflict. Even under conditions of local and fuzzy information about these motives, alliances exhibit this effect.

The computational model lends credibility to a mechanism that is difficult, if not impossible, to subject to a general empirical test. So far, the evidence we have is anecdotal and insufficient to allow for a systematic analysis. A model such as the one presented here serves to establish a lower boundary on the effect; although it is strongly biased towards the local level and features behaviorally simple agents, we observe a violence-promoting effect of alliances. In more realistic context, this effect is likely to be stronger. These conclusions are obviously based on an abstract model, and applications to particular cases - let alone particular instances of violence - are difficult, largely owing to the empirical measurement problems discussed above. Still, computational approaches of this kind help to make "soft" arguments about civil war operational, and can provide opportunities for testing their implications and for further exploration.

\section{Acknowledgments}

The author gratefully acknowledges feedback from Margit Bussmann and Martin Nome on an earlier version of this paper.

\section{Funding}

This research was partly funded by the Alexander von Humboldt Foundation (Sofja Kovalevskaja Award).

\section{Supplementary material}

Model source code for the NetLogo modeling package is available from the author's DataVerse page (http://dx.doi.org/10.7910/DVN/28444), along with the Online Appendix. 


\section{Notes}

1. The small-world network creation algorithm starts by assigning agents to a set of "villages" with a high density of intra-village connections, and then randomly rewiring a certain proportion of these intra-village links to form links between villages. Thus, two parameters guide the creation of a network: first, the size of the initial villages (the radius); and second, the rewiring probability. The latter controls the extent to which the network remains rural (low rewiring probability) or turns into an urban one (high probability), and is what we call the urbanization parameter in the model.

2. Rather than fixing each run to 1000 steps, an alternative could have been to terminate runs when some sort of convergence has been reached. However, since the model is volatile in the sense that the rate of violence can still vary between runs, convergence is difficult to detect from within the simulation. Therefore, it is preferable to set a uniform time limit for all simulation runs, which gives the model sufficient time to settle to a stable state.

\section{References}

Axelrod R (1997) The dissemination of culture. Journal of Conflict Resolution 41(2): 203-226.

Buhaug H and Gates S (2002) The geography of civil war. Journal of Peace Research 39(4): 417-433.

Cederman L-E (2001) Agent-based modeling in political science. The Political Methodologist 10: 16-22.

Cederman L-E (2003) Modeling the size of wars: From billiard balls to sandpiles. American Political Science Review 97(1): 135-150.

Cederman L-E and Gleditsch KS (2009) Introduction to special issue on "Disaggregating Civil War". Journal of Conflict Resolution 53(4): 487-495.

Cederman L-E, Wimmer A and Min B (2010) Why do ethnic groups rebel? New data and analysis. World Politics 62(1): 87-119.

Collier P and Hoeffler A (2004) Greed and grievance in civil war. Oxford Economic Papers 56(4): $563-595$.

Fearon JD and Laitin DD (2003) Ethnicity, insurgency and civil war. American Political Science Review 97(1): 75-90.

Fujii LA (2009) Killing Neighbors: Webs of Violence in Rwanda. Ithaca, NY: Cornell University Press.

Gilbert N (2007) Agent-based Models. London: Sage.

Hegre H, Østby G and Raleigh C (2009) Poverty and civil war events: A disaggregated study of Liberia. Journal of Peace Research 53(4): 598-623.

Kalyvas SN (2003) The ontology of political violence: Action and identity in civil wars. Perspectives on Politics 1(3): 475-494.

Kalyvas SN (2006) The Logic of Violence in Civil War. New York: Cambridge University Press.

Laver M and Sergenti E (2007) Do ideologically intransigent parties affect the policy positions of other parties? And ... rigorously characterizing output from computational models of party competition. Paper prepared for the Annual Meeting of the American Political Science Association.

Lustick IS, Miodownik D and Eidelson RJ (2004) Secessionism in multicultural states: Does sharing power prevent or encourage it? American Political Science Review 98(2): 209-229.

Mueller J (2000) The banality of ethnic war. International Security 25(1): 42-70.

Raleigh C and Hegre H (2009) Population size, concentration and civil war: A geographically disaggregated analysis. Political Geography 28(4): 224-238.

Raleigh C, Linke A, Hegre H and Karlsen J (2010) Introducing ACLED: An armed conflict location and event dataset. Journal of Peace Research 47(5): 651-660.

Salehyan I (2010) The delegation of war to rebel organizations. Journal of Conflict Resolution 54(3): 493-515.

Schelling TC (1978) Micromotives and Macrobehavior. New York: Norton.

Starr H (1978) "Opportunity" and "willingness" as ordering concepts in the study of war. International Interactions 4: 363-387. 
Sundberg R and Melander E (2013) Introducing the UCDP geo-referenced event dataset. Journal of Peace Research 50(4): 523-532.

Watts DJ and Strogatz SH (1998) Collective dynamics of "small-world" networks. Nature 393: $440-442$.

Weidmann NB (2011) Violence "from above" or "from below"? The role of ethnicity in Bosnia's civil war. Journal of Politics 73(4): 1178-1190.

Weidmann NB and Ward MD (2010) Predicting conflict in space and time. Journal of Conflict Resolution 54(6): 883-901.

Wilensky U (1999) NetLogo. Agent-based modeling software. Center for Connected Learning and Computer-Based Modeling, Northwestern University. Evanston, IL. Available at: http:// ccl.northwestern.edu/netlogo/ 